\title{
The Structure of EAP-Groups and Self-Autopermutable Subgroups
}

\author{
Shima Housieni ${ }^{1}$ and Mohammad Reza Rajabzadeh Moghaddam ${ }^{1,2,3}$ \\ ${ }^{1}$ Department of Mathematics, Islamic Azad University, Mashhad Branch, Mashhad 9187147578, Iran \\ ${ }^{2}$ Department of Mathematics, Khayyam University, Mashhad 9189747178, Iran \\ ${ }^{3}$ Centre of Excellence in Analysis on Algebraic Structures, Ferdowsi University of Mashhad, P.O. Box 1159-91775, Iran
}

Correspondence should be addressed to Mohammad Reza Rajabzadeh Moghaddam; rezam@ferdowsi.um.ac.ir

Received 19 May 2014; Accepted 16 September 2014; Published 11 December 2014

Academic Editor: K. C. Sivakumar

Copyright (C) 2014 S. Housieni and M. R. R. Moghaddam. This is an open access article distributed under the Creative Commons Attribution License, which permits unrestricted use, distribution, and reproduction in any medium, provided the original work is properly cited.

\begin{abstract}
A subgroup $H$ of a given group $G$ is said to be autopermutable, if $H H^{\alpha}=H^{\alpha} H$ for all $\alpha \in \operatorname{Aut}(G)$. We also call $H$ a selfautopermutable subgroup of $G$, when $H H^{\alpha}=H^{\alpha} H$ implies that $H^{\alpha}=H$. Moreover, $G$ is said to be EAP-group, if every subgroup of $G$ is autopermutable. One notes that if $\alpha$ runs over the inner automorphisms of the group, one obtains the notions of conjugatepermutability, self-conjugate-permutability, and ECP-groups, which were studied by Foguel in 1997, Li and Meng in 2007, and Xu and Zhang in 2005, respectively. In the present paper, we determine the structure of a finite EAP-group when its centre is of index 4 in $G$. We also show that self-autopermutability and characteristic properties are equivalent for nilpotent groups.
\end{abstract}

\section{Introduction}

Let $H$ be a subgroup of a given group $G$. Then we call $H$ to be autopermutable, if $H H^{\alpha}=H^{\alpha} H$ for all $\alpha \epsilon$ $\operatorname{Aut}(G)$. The subgroup $H$ is said to be self-autopermutable, if $H H^{\alpha}=H^{\alpha} H$ implies that $H^{\alpha}=H$. Moreover, we call the group $G$ to be an EAP-group if every subgroup of $G$ is autopermutable. Clearly, if $\alpha$ runs over the inner automorphisms of the group, we obtain the notions of conjugatepermutability [1], self-conjugate-permutability [2], and ECPgroups [3], respectively. One notes that the subgroup $H=$ $\langle b\rangle$ of the Dihedral group $D_{8}=\left\langle a, b: a^{4}=b^{2}=1, a^{b}=a^{-1}\right\rangle$ is conjugate-permutable, which is not autopermutable. To see this, consider the automorphism $\alpha$ which sends $a$ and $b$ into $a$ and $a b$, respectively. Clearly $H H^{\alpha}$ is not a subgroup of $G$, which means that $H H^{\alpha} \neq H^{\alpha} H$. It is easily seen that similar examples can be obtained by taking a direct product of $D_{8}$ with any other group. Also, every noncharacteristic normal subgroup of a given group is an example for a self-conjugate-permutable subgroup which is not selfautopermutable. Moreover, $D_{8}$ is an ECP-group, which is not an EAP-group.
In the present paper, we determine the structure of a finite EAP-group, when its centre is of index 4 . We also prove that self-autopermutability and characteristic properties are equivalent in nilpotent groups.

\section{Finite EAP-Groups}

In this section, we determine the structure of finite EAPgroups, when their centres are of index 4 . In fact we prove the following theorem.

Theorem 1. Let $G$ be a finite group with the centre of index 4 . Then $G$ is an EAP-group if and only if the Sylow 2-subgroup of $G$ is one of the following forms:

(i) $Q_{8}$;

(ii) $\left\langle a, b \mid a^{2^{n+1}}=b^{2}=1, a^{b}=a^{2^{n}+1}\right\rangle, n \geq 3$;

(iii) $\mathbb{Z}_{2} \times Q_{8}$;

(iv) $\langle a, b, c, d| a^{2}=b^{2}=c^{4}=d^{4}=1, b \in Z(G),[a, c]=$ $\left.[a, d]=c^{3} d,[c, d]=1\right\rangle$; 
(v) $\langle a, b, c, d, e| a^{2}=b^{2}=c^{2}=d^{2}=e^{4}=1, a, b, c \in$ $Z(G),[d, e]=b\rangle$;

(vi) $\langle a, b, c, d, e| a^{2}=b^{2}=c^{2}=d^{4}=e^{4}=1, a, b, c \in$ $Z(G),[d, e]=a\rangle$.

We remind that a nonabelian group is said to be Hamiltonian, if all of its subgroups are normal. The following result gives our claim, when $G$ is a 2-group with cyclic centre of index 4.

Theorem 2. Let $G$ be a finite 2-group with cyclic centre of index 4. Then $G$ is an EAP-group if and only if $G \cong Q_{8}$ or $\langle a, b|$ $\left.a^{2^{n+1}}=b^{2}=1, a^{b}=a^{2^{n}+1}\right\rangle$, for all $n \geq 3$.

Proof. Consider the group $G$ to be $Q_{8}$. Since $Q_{8}$ is Hamiltonian group, the result follows easily. Now assume $G=\langle a, b|$ $\left.a^{2^{n+1}}=b^{2}=1, a^{b}=a^{2^{n}+1}\right\rangle, n \geq 3$. One can easily check that $G$ contains exactly three proper subgroups of orders $2^{i}$, for $1 \leq i \leq n+1$. We also observe that the subgroups of orders 2 are autopermutable and as the subgroups of orders $2^{n+1}$ are normal, they are also autopermutable. Now, one can check that there are exactly two cyclic and one noncyclic subgroups of orders $2^{i}, 2 \leq i \leq n$, so that one of the cyclic subgroups is central and hence all the subgroups of $G$ satisfy the required property.

Conversely, assume that $G$ is an EAP-group, $Z(G)=\langle x|$ $\left.x^{2^{n}}=1\right\rangle, G / Z(G)=\{Z(G), a Z(G), b Z(G), a b Z(G)\}$, where $a^{2}, b^{2} \in Z(G)$ and so $|a|,|b| \leq 2^{n+1}$. In case $n=1$, then the group $G$ is either $D_{8}$ or $Q_{8}$. As explained before, $D_{8}$ cannot be an EAP-group and hence $G \cong Q_{8}$. Now suppose $n>1$ and the elements $a$ and $b$ are both of order 2. Then every element $y \in G$ has the following form (as $G$ is nilpotent of class 2):

$$
y=a^{i} b^{j} x^{k}, \quad \text { where } 0 \leq i, j<2,0 \leq k<2^{n} .
$$

Clearly, the map $\alpha$ given by $\alpha(y)=b^{i} a^{j} x^{k}$ is an automorphism of $G$, which sends $a$ into $b$. Thus $H H^{\alpha} \neq H^{\alpha} H$ for the subgroup $H=\langle b\rangle$, which contradicts the assumption. Now, if $|a|,|b|<2^{n+1}$ we may replace $a$ and $b$ by the elements $a x^{i}$ and $b x^{j}$, both of which are of order 2 . This reduces to the previous case. Therefore we must have $a$ or $b$ of order $2^{n+1}$. Then $G$ has a cyclic subgroup of order $2^{n+1}$ and so $G$ is of order $2^{n+2}(n>1)$ with the centre of index 4 . Hence, by $[4,5.3 .4]$, the group $G$ has the following presentation:

$$
G=\left\langle a, b \mid a^{2^{n+1}}=b^{2}=1, a^{b}=a^{2^{n}+1}\right\rangle, \quad n \geq 3 .
$$

This is an EAP-group and so the proof is completed.

The following result considers the case when $G$ is a 2-group with noncyclic centre of index 4.

Theorem 3. Let $G$ be a finite 2-group with noncyclic centre of index 4. Then $G$ is an EAP-group if and only if $G$ is one of the following forms:

(i) $G=\mathbb{Z}_{2} \times Q_{8}$;

(ii) $G=\langle a, b, c, d| a^{2}=b^{2}=c^{4}=d^{4}=1, b \in$ $\left.Z(G),[a, c]=[a, d]=c^{3} d,[c, d]=1\right\rangle ;$ (iii) $G=\langle a, b, c, d, e| a^{2}=b^{2}=c^{2}=d^{2}=e^{4}=1, a, b, c \in$ $Z(G),[d, e]=b\rangle$;

(iv) $G=\langle a, b, c, d, e| a^{2}=b^{2}=c^{2}=d^{4}=e^{4}=1, a, b, c \in$ $Z(G),[d, e]=a\rangle$.

Proof. The sufficient condition is obvious. We only need to prove the necessity condition. Let $G$ be an EAP-group and $G / Z(G)=\{Z(G), a Z(G), b Z(G), a b Z(G)\}$, where $a^{2}, b^{2} \in$ $Z(G)$. Assume that $Z(G)$ is not an elementary abelian 2group. Since $Z(G)$ is the direct product of its cyclic subgroups, by the same argument as in Theorem 2, there are no EAPgroups in this case. Now, assume that $Z(G)$ is an elementary abelian 2-group. Clearly $G$ must be a group of order either 16 or 32. The structure of such groups is given as follows in [5]. If $|G|=16$, then

(i) $G=\mathbb{Z}_{2} \times D_{8}$;

(ii) $G=\left\langle a, b \mid a^{4}=b^{4}=1, b a b^{-1}=a^{3}\right\rangle \cong \mathbb{Z}_{4} \rtimes \mathbb{Z}_{4}$;

(iii) $G=\mathbb{Z}_{2} \times Q_{8}$;

(iv) $G=\langle a, b, c, d| a^{2}=b^{2}=c^{4}=d^{4}=1, b \epsilon$ $\left.Z(G),[a, c]=[a, d]=c^{3} d,[c, d]=1\right\rangle$.

As $D_{8}$ is not an EAP-group, hence the group of form (i) cannot be an EAP-group. For the group of form (ii) we can consider $H=\langle b\rangle$ and $\alpha \in \operatorname{Aut}(G)$ which sends $a$ and $b$ into $a$ and $a b$, respectively. Clearly, $H H^{\alpha} \neq H^{\alpha} H$ and hence $G$ cannot be an EAP-group. Thus when $|a|=|b|$, then $G$ is of the form given in either (iii) or (iv).

Assume $|G|=32$. Then such groups in the list of small groups with elementary abelian centres of index 4 are only of the following forms:

(i) $G=\langle a, b, c| a^{4}=b^{4}=c^{2}=1,[a, b]=c,[a, c]=$ $[b, c]=1\rangle$;

(ii) $G=\langle a, b, c, d, e| a^{2}=b^{2}=c^{2}=d^{4}=e^{4}=1, a, b, c \in$ $Z(G),[d, e]=b\rangle$;

(iii) $G=\langle a, b, c, d, e| a^{2}=b^{2}=c^{2}=d^{2}=e^{2}=1, c, d \in$ $Z(G),[a, e]=[b, e]=a b,[a, b]=1\rangle ;$

(iv) $G=\langle a, b, c, d, e| a^{2}=b^{2}=c^{2}=d^{2}=e^{4}=1, a, b, c \in$ $Z(G),[d, e]=b\rangle$;

(v) $G=\langle a, b, c, d, e| a^{2}=b^{2}=c^{2}=d^{4}=e^{4}=1, a, b, c \in$ $Z(G),[d, e]=a\rangle$.

For the group of form (i) we may consider the cyclic subgroup $H=\langle b\rangle$ and $\alpha \in \operatorname{Aut}(G)$, which sends $a, b$, and $c$ into $a, a b$, and $c$, respectively. In case the group $G$ is of form (ii), we consider $H=\langle e\rangle$ and $\alpha \in \operatorname{Aut}(G)$ which sends $a$, $b, c, d$, and $e$ into $a, a b, a c, b e$, and $e d$, respectively. Also if the group is considered to be of form (iii), one may consider $H=\langle e\rangle$ and $\alpha \in \operatorname{Aut}(G)$ which sends $a, b, c, d$, and $e$ into $e$, $b, c, d$, and $a$, respectively. Now, one can easily check that in these cases $H H^{\alpha} \neq H^{\alpha} H$ and so $G$ cannot be an EAP-group. Hence, when $|G|=32$, then $G$ is of either form (iv) or form (v). The proof is complete.

Proof of Theorem 1. The necessity condition is obvious and Theorems 2 and 3 establish the result, when $G$ is a 2-group. If $G$ is not a 2-group, then we may write $G=S_{1} \times S_{2} \cdots \times S_{k}$, 
in such a way that $S_{1}$ is a Sylow 2-subgroup and $S_{i}$ is an abelian Sylow $p_{i}$-subgroup, where $p_{i}$ is an odd prime number, for $2 \leq i \leq k$. Clearly, $\operatorname{Aut}(G) \cong \operatorname{Aut}\left(S_{1}\right) \times \operatorname{Aut}\left(S_{2}\right) \times \cdots \times \operatorname{Aut}\left(S_{k}\right)$ and for any subgroup $H$ of $G, H \cong H_{1} \times H_{2} \times \cdots \times H_{k}$, where $H_{i} \leq S_{i}$ for $1 \leq i \leq k$. Thus $H$ is an autopermutable subgroup of $G$ if $H_{1}$ is an autopermutable subgroup of $S_{1}$. This completes the proof.

\section{Self-Autopermutable Subgroups in Nilpotent Groups}

We call a subgroup $H$ of a given group $G$ to be weakly characteristic, when $H^{\alpha} \leq N_{G}(H)$ implies that $H^{\alpha}=H$ for all $\alpha \in \operatorname{Aut}(G)$. Also, given the subgroups $H$ and $K$, then $H$ satisfies the subcharacteriser condition, if $H \unlhd K$ implies that $N_{\text {Aut }(G)}(K) \leq N_{\text {Aut }(G)}(H)$, where $N_{\text {Aut }(G)}(K)=\{\alpha \in$ $\left.\operatorname{Aut}(G) ; K^{\alpha}=K\right\} \leq \operatorname{Aut}(G)$. Clearly, if one considers the inner automorphisms of the group then weakly normal and normaliser condition properties are obtained.

The following result of [6] shows that self-conjugatepermutability, weakly normal property, and subnormaliser condition are equivalent for $p$-subgroups of a given group.

Theorem 4 (see [6], Proposition 3.3). Let $H$ be a p-subgroup of a group $G$. Then the following properties are equivalent:

(i) $H$ is a self-conjugate-permutable subgroup;

(ii) $H$ is a weakly normal subgroup;

(iii) H satisfies the subnormaliser condition.

In this section, it is shown that self-autopermutable subgroups in nilpotent groups are always characteristic.

Proposition 5. Let $H$ be a subgroup of a group $G$.

(i) If $H$ is self-autopermutable, then $H$ is weakly characteristic in $G$.

(ii) If $H$ is weakly characteristic, then $H$ satisfies the subcharacteriser condition in $G$.

Proof. (i) If $H^{\alpha} \leq N_{G}(H)$, as $H \unlhd N_{G}(H)$, we have $H H^{\alpha}=$ $H^{\alpha} H$. Applying the condition that $H$ is self-autopermutable subgroup of the group $G$, we get $H^{\alpha}=H$. By definition, $H$ is weakly characteristic.

(ii) Let $K \leq G$, such that $H \unlhd K$. We have $H^{\alpha} \leq K^{\alpha}=$ $K \leq N_{G}(H)$ for every $\alpha \in N_{\text {Aut }(G)}(K)$. Since $H$ is weakly characteristic in $G$, we have $H^{\alpha}=H$. Thus $\alpha \in N_{\operatorname{Aut}(G)}(H)$ and the result is obtained.

The following theorem is one of the main results in this section.

Theorem 6. Let $H$ be a subgroup of a nilpotent finite group $G$. If $H$ satisfies the subcharacteriser condition then $H$ is characteristic in $G$.

Proof. Write $G \cong P_{1} \times P_{2} \times \cdots \times P_{t}$, where $P_{i}$ is a Sylow $p_{i}$-subgroup of $G$, for $1 \leq i \leq t$. We may also write $H \cong$ $H_{1} \times H_{2} \times \cdots \times H_{t}$, with $H_{i} \leq P_{i}, 1 \leq i \leq t$. Since $H$ satisfies the subcharacteriser condition in $G$, one can easily see that $H_{i}$ satisfies the subcharacteriser condition in $P_{i}$. Therefore $H_{i} \unlhd P_{i}$ implies that $H_{i}$ is characteristic in $P_{i}$, which proves the result.

Finally, we show that self-autopermutability, weakly characteristic, and subcharacteriser conditions are equivalent, for every subgroup of a nilpotent group.

Corollary 7. Let $H$ be a subgroup of a finite nilpotent group G. Then

(i) $H$ is a self-autopermutable;

(ii) $H$ is a weakly characteristic;

(iii) H satisfies the subcharacteriser condition in $G$.

Proof. The result follows by Proposition 5 and Theorem 6.

\section{Conflict of Interests}

The authors declare that there is no conflict of interests regarding the publication of this paper.

\section{References}

[1] T. Foguel, “Conjugate-permutable subgroups," Journal of Algebra, vol. 191, no. 1, pp. 235-239, 1997.

[2] S. Li and Z. Meng, "Groups with conjugate-permutable conditions," Mathematical Proceedings of the Royal Irish Academy, vol. 107, no. 2, pp. 115-121, 2007.

[3] M. Xu and Q. Zhang, "On conjugate-permutable subgroups of a finite group," Algebra Colloquium, vol. 12, no. 4, pp. 669-676, 2005.

[4] D. J. Robinson, A Course in the Theory of Groups, Springer, New York, NY, USA, 2nd edition, 1996.

[5] The GAP Group, "Algorithms and Programming," Version 4.4.12, 2008, http://www.gap-system.org.

[6] Z. Shen, W. Liu, and X. Kong, "Finite groups with self-conjugate permutable subgroups," Communications in Algebra, vol. 38, no. 5, pp. 1715-1724, 2010. 


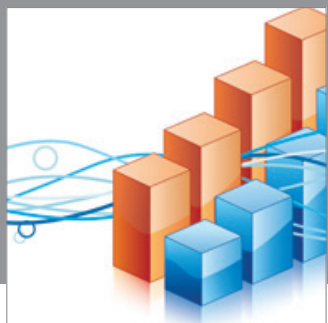

Advances in

Operations Research

mansans

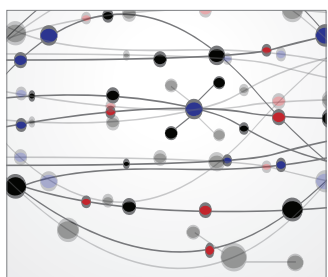

The Scientific World Journal
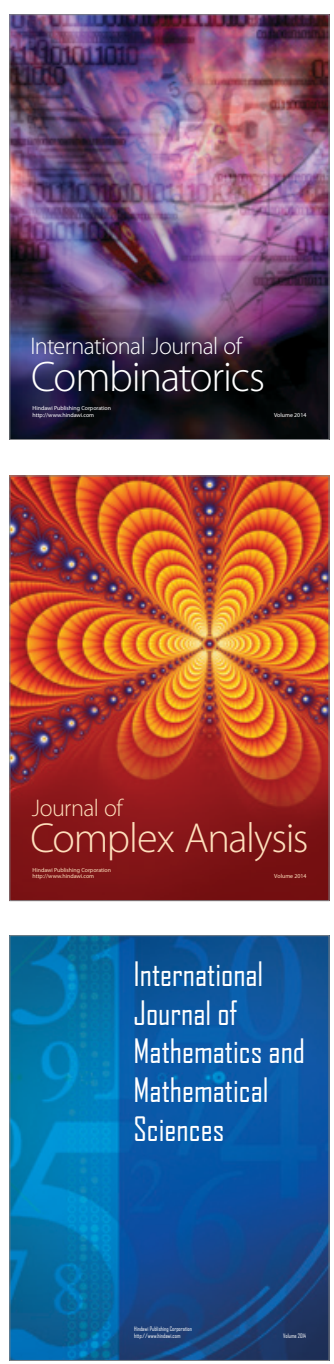
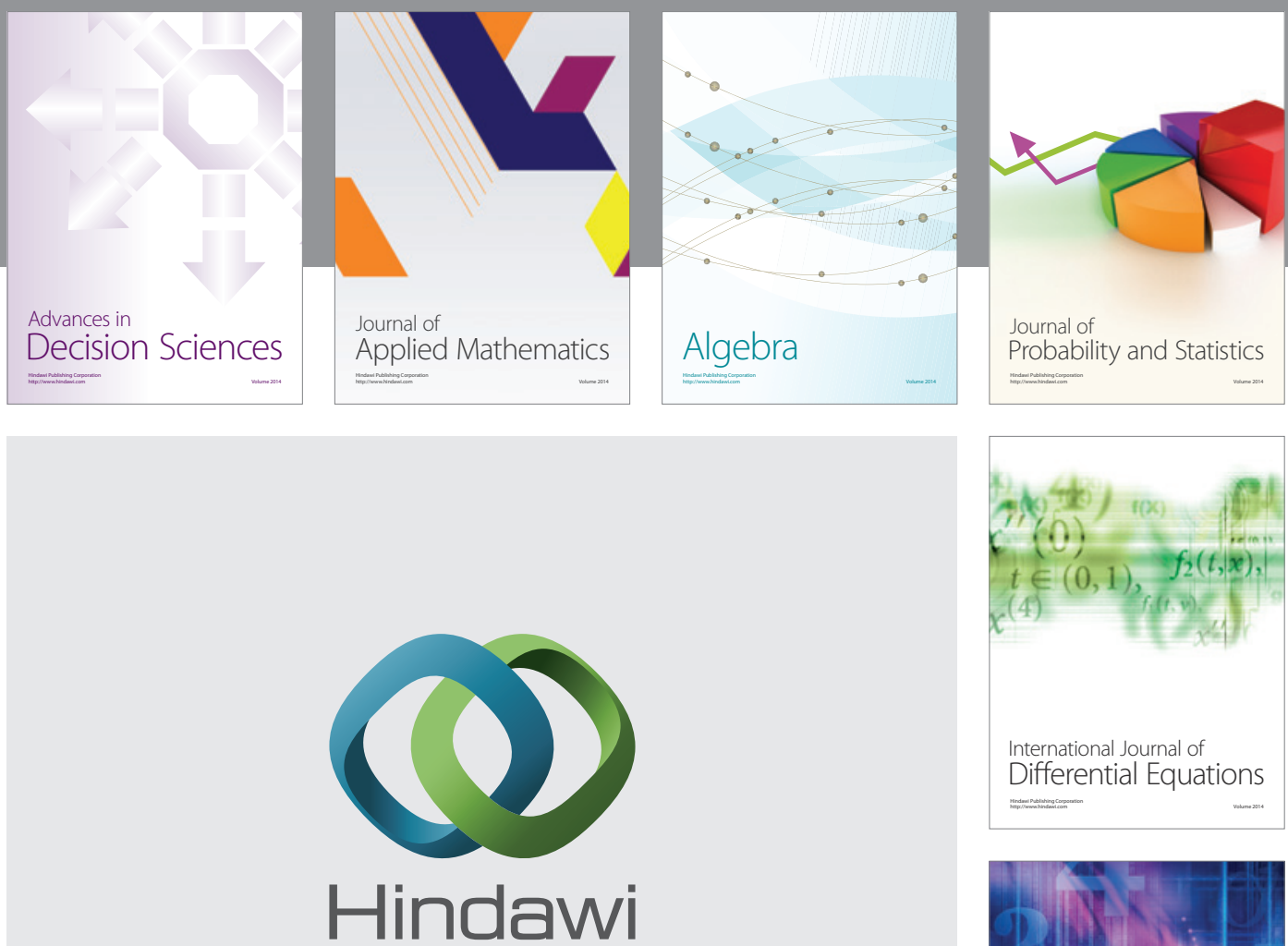

Submit your manuscripts at http://www.hindawi.com
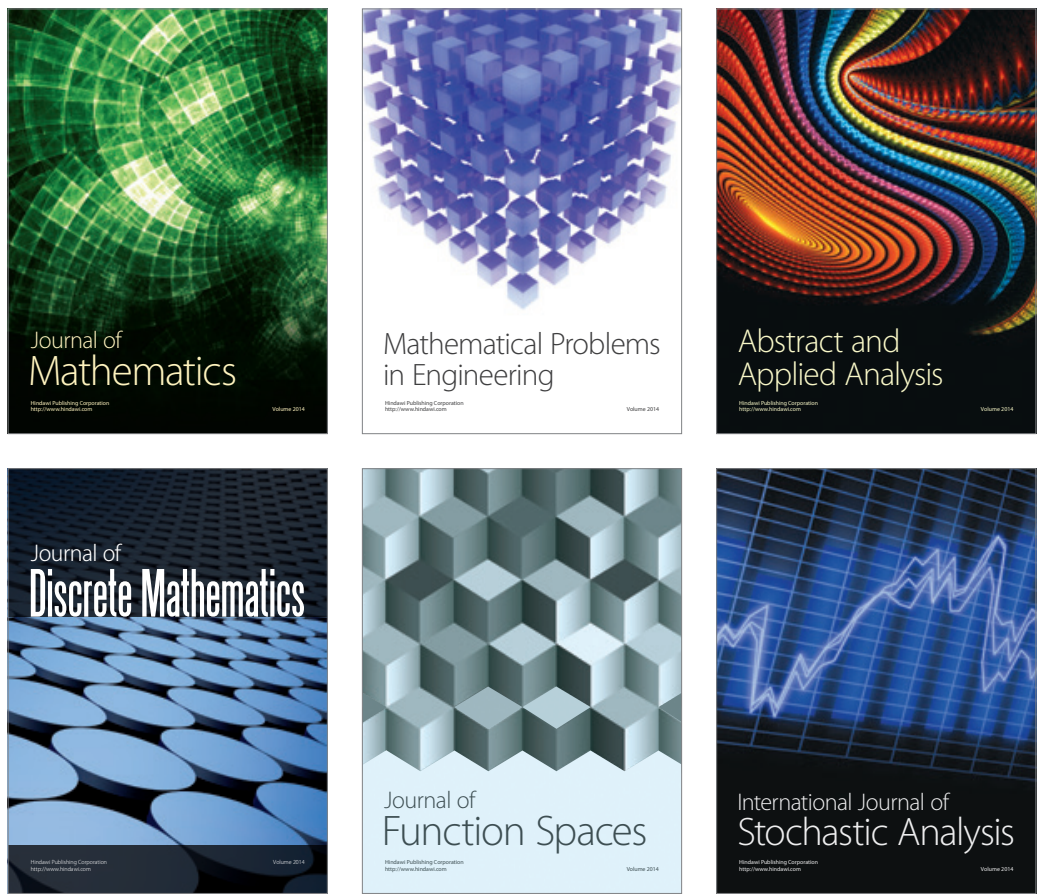

Journal of

Function Spaces

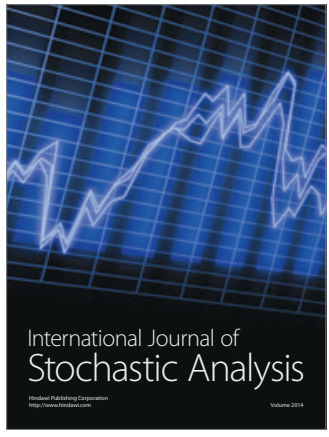

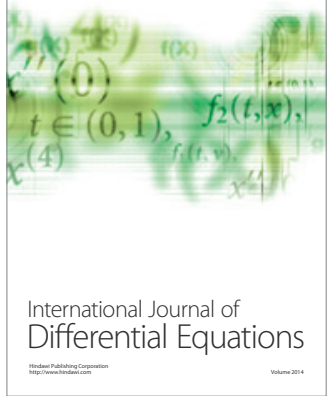
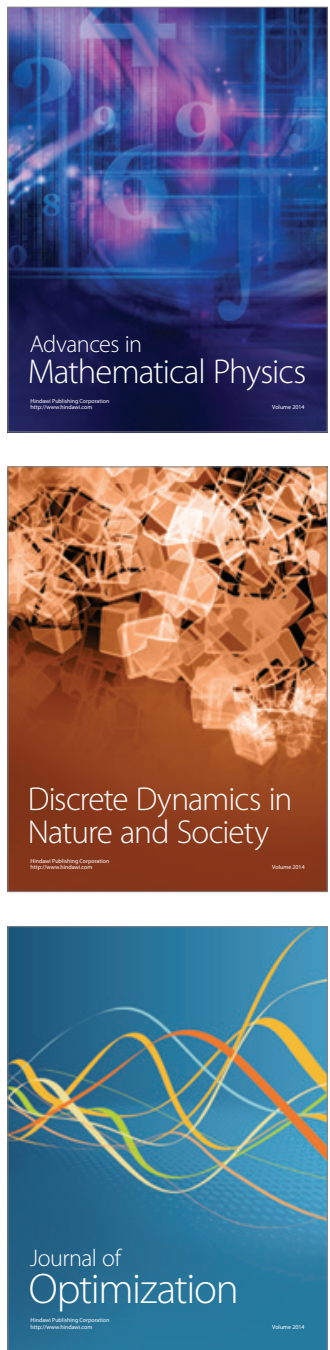